\title{
Rugoscopia palatina e seus desafios na identificação humana: uma revisão
}

\section{integrativa}

\author{
Palatine rugoscopy and its challenges in human identification: an integrative review \\ La rugoscopia palatina y sus desafíos en la identificación humana: una revisión integrativa
}

Recebido: 20/03/2021 | Revisado: 26/03/2021 | Aceito: 29/03/2021 | Publicado: 09/04/2021

\author{
Isabelle Silva Ramos das Neves \\ ORCID: https://orcid.org/0000-0001-5078-1210 \\ Universidade Federal de Pernambuco, Brasil \\ E-mail: isabellenevesodontologia@gmail.com \\ Adriana Paula de Andrade da Costa e Silva Santiago \\ ORCID: https://orcid.org/0000-0003-0250-0204 \\ Universidade Federal de Pernambuco, Brasil \\ E-mail: adriana.acsilva@ufpe.br \\ Maria Izabel Teixeira da Silva \\ ORCID: https://orcid.org/0000-0001-8097-9682 \\ Universidade Federal de Pernambuco, Brasil \\ E-mail: izabelteixeira33@gmail.com \\ Evellyn Rayane Martins de Oliveira \\ ORCID: https://orcid.org/0000-0002-6351-6528 \\ Universidade Federal de Pernambuco, Brasil \\ E-mail: evellynolii84@gmail.com
}

\begin{abstract}
Resumo
Esta revisão integrativa teve por objetivo verificar como a identificação da Odontologia Legal, por meio das rugas palatinas, tem se apresentado na literatura científica e quais as limitações para o seu uso em perícias post mortem. O que demandou em uma busca sob os seguintes descritores: Rugoscopia palatina, Palatoscopia e Odontologia forense, nas bases de dados da PubMed, BVS (Medline e Lilacs), BBO e Scielo, para artigos publicados entre 2010 e 2019. Foram selecionados os que se apresentavam disponíveis com texto completo e gratuito, em idiomas de inglês e português, cuja conclusão estivesse de acordo com o propósito deste estudo, deixando de fora os artigos que tratassem de estudos relacionados a perícia no vivo ou ante mortem, bem como os repetidos. Foram contabilizados um total de 58 artigos, sendo que destes, 7 artigos atendiam por completo aos critérios de inclusão/exclusão. Estes tratavam de revisões de literatura narrativa, sistemática e meta-análise, relatos de caso pericial e revisão de relatos de caso. Podese concluir que a rugoscopia palatina se apresenta na literatura científica como um método útil como guia na identificação humana, normalmente complementar, e ainda com possibilidade de transmitir diversas informações. Porém, não deve ser utilizada como único método de identificação da vítima, posto que também apresenta várias limitações, as quais podem dificultar ou mesmo inviabilizar o procedimento identificatório.
\end{abstract}

Palavras-chave: Palatoscopia; Rugoscopia palatina; Odontologia Forense.

\begin{abstract}
This integrative review aimed to verify how the identification of Forensic Dentistry, by means palatal wrinkles, has been presented in the scientific literature and which are the limitations for its use in post mortem forensics. That required a search under the following descriptors: Palatal Rugoscopy, Palatoscopy and Forensic Dentistry, in the databases of Pubmed, BVS (Medline and Lilacs), BBO and Scielo for published articles among 2010 to 2019. Those that were available with full and free text in Portuguese and English languages whose conclusion was according to the purpose of this study, leaving out the articles related to live and ante mortem forensics, as well as the repeated ones. From a total of 58 Articles counted, 7 met fully the inclusion /exclusion criteria. These articles puntuacted reviews of narrative, sistematic literature and meta-analysis, forensic case reports and review of these cases. It can be concluded that Palatal Rugoscopy has been presented in the scientific literature as a useful guide in human identification, applied, usually, as a complementary method, but still can provide many additional information; However, should not be used as the only method of identifying the victim, once that also has many noted limitations that can difficult or even to make the identification unfeasible.
\end{abstract}

Keywords: Palatoscopy; Palatal rugoscopy; Forensic dentistry.

\section{Resumen}

Esta revisión integradora tuvo como objetivo verificar cómo la identificación de la Odontología Forense, através de las arrugas palatinas, se ha presentado en la literatura científica y cuáles son las limitaciones para su uso en la pericia post-mortem. Lo que exigió en una búsqueda bajo los siguientes descriptores: Rugoscopia palatina, Palatoscopia y 
odontología forense, en las bases de datos de PubMed, BVS (Medline y Lilacs), BBO y Scielo, para artículos publicados entre 2010 y 2019. Se seleccionaron los que estuvieron disponibles en texto completo y libre, en inglés y portugués, cuya conclusión fue acorde con el propósito de este estudio, dejando de lado los artículos que trataban de estudios relacionados con la pericia en vivo o ante-mortem, así como los repetidos. Se contabilizaron un total de 58 artículos, de estos, 7 artículos cumplieron plenamente los criterios de inclusión / exclusión. Se trataba de revisiones de literatura narrativa, sistemática y de metanálisis, informes de casos de expertos y revisión de informes de casos. Se puede concluir que la rugoscopia palatina se presenta en la literatura científica como un método útil como guía en la identificación humana, generalmente complementaria, y con posibilidad de transmitir información diversa. Sin embargo, no debe utilizarse como el único método de identificación de la víctima, ya que también tiene varias limitaciones, que pueden dificultar o incluso hacer inviable el procedimiento de identificación.

Palabras clave: Palatoscopia; Rugoscopia palatina; Odontología forense.

\section{Introdução}

O processo cuja finalidade é levantar uma identidade chama-se identificação, ela pode ser realizada por técnicos treinados ou por profissionais com conhecimentos diferenciados e específicos, e consiste na comparação dos caracteres, procurando as coincidências entre os dados previamente registrados e os obtidos no presente. E a identificação pessoal pode ser realizada tanto, em indivíduos vivos quanto após o óbito, em suas mais diversas condições (conservados, em decomposição, carbonizados, esquartejados, mutilados e fragmentados) (Tornavoi \& Silva, 2010).

Modesto e Junior (2017) afirmam, que a identificação humana tem grande importância na Medicina Forense, tanto por razões legais como humanas, sendo frequentemente iniciada antes mesmo de se determinar a causa da morte do indivíduo. A identificação de um cadáver tanto sob o aspecto civil, além do aspecto social, também se reveste do aspecto legal, na medida em que um processo judicial, como autorização para sepultamento de uma pessoa da família, vem a trazer conforto para os demais membros familiares.

No entanto, para um processo de identificação ser considerado confiável, os métodos utilizados nesta atuação devem seguir três parâmetros biológicos e duas características técnicas, respectivamente: unicidade ou individualidade (onde o que está sendo estudado apresenta características únicas e diferentes dos demais indivíduos), perenidade (onde as características resistem a ação do tempo), imutabilidade (onde as características não se alteram com o passar do tempo), praticabilidade (o método precisa ser de fácil aplicação e de baixo custo), e classificabilidade (facilita o armazenamento e a busca de dados dos registros das características de um indivíduo) (Santos, Fernandes, \& Serra, 2011; Miranda et al., 2011; Castro-Silva, Silva, \& Veiga, 2014; Junior \& Moura, 2014; Alves \& Vasconcelos, 2017; Modesto \& Junior, 2017; Garbin, Amaral, \& Greghi, 2017; Correia et al., 2019; Gomes et al., 2019).

Dentro da ciência forense, os métodos mais comumente utilizados no processo de identificação humana são: análise do ácido desoxirribonucleico (DNA), da impressão digital (através do sistema de Vucetich), e do arco dentário (técnica de identificação post-mortem). Porém, há restrições no uso destes métodos. A análise do DNA é um dos métodos mais onerosos, e, devido a isso, pouco utilizado em situações onde se pode lançar mão de outros procedimentos. Nas impressões digitais essas restrições, como situações de carbonizações ou mutilações que atingem as mãos do indivíduo se tornam impossíveis, pois necessitam do registro das linhas digitais. Em relação aos arcos dentários, embora os dentes sejam mais duráveis, não é prático empregá-los na identificação de pessoas desdentadas. Neste caso, o método da rugoscopia palatina, utilizado pelos odontolegistas, se torna alternativa útil para a identificação de um indivíduo (Adisa, Kolude, \& Ogunrinde, 2014; Olivo, Ricardo, \& Garcia, 2014; Lima et al., 2016; Babaji, Jalal, \& Kamalaksharappa, 2018; Correia et al., 2019).

A rugoscopia palatina é um estudo sistemático ou técnico de identificação que estuda, classifica e registra as rugas palatinas com o objetivo de contribuir no processo de identificação humana de casos complexos, onde não conseguimos identificar através de outros métodos convencionais (Eboh, 2012; Barbieri et al., 2013; Matute, 2015), e promovem a individualização através da forma, tamanho e posição das pregas/rugas ou cristas palatinas que se encontram na parte anterior 
da mucosa do palato, sendo possível sua aplicação na identificação tanto no cadáver recente como no indivíduo vivo (Oliveira \& Marques, 2015).

Isto considerado, este estudo teve como objetivo responder a seguinte questão norteadora: “Como a identificação da Odontologia Legal, por meio das rugas palatinas, tem se a apresentado na literatura científica e quais as dificuldades para o seu uso em perícias de identificação post mortem?”, por meio de uma revisão integrativa de literatura.

\section{Metodologia}

Esta revisão do tipo integrativa foi baseada na proposta de Whittemore e Knafl (2005), de onde foram seguidas as etapas descritas a seguir:

$>\quad$ Etapa 1: foram feitas a identificação da questão do estudo ou problematização e as buscas pelos descritores ou palavras-chave (Rugoscopia Palatina, Palatoscopia e Odontologia Forense, em inglês ou português) nas bases de dados da PubMed, Portal Regional da BVS, especificamente na MedLine, Lilacs e BBO, e Scielo. Para orientar o presente estudo buscou-se responder a seguinte pergunta: Como a identificação da Odontologia Legal, por meio das rugas palatinas, tem se a apresentado na literatura científica e quais as limitações para o seu uso em perícias de identificação post mortem?

$>$ Etapa 2: foram realizadas a seleção da amostra e a verificação dos critérios de inclusão e exclusão, estabelecendo melhor qualidade e confiabilidade na seleção (Quadro 1).

Quadro 1. Definição dos critérios de inclusão e exclusão da literatura pesquisada.

\begin{tabular}{|l|l|}
\hline \multicolumn{1}{|c|}{ Critérios de inclusão } & \multicolumn{1}{c|}{ Critérios de exclusão } \\
\hline a) Artigo publicado no período de janeiro de 2010 até & a) Artigos publicados antes do período delimitado \\
dezembro de 2019; & que estivessem em outros idiomas; \\
b) Texto completo e gratuito disponível; & b) Estudos relacionados a perícias no vivo ou ante- \\
c) Publicados em português, inglês e espanhol; & mortem; \\
d) Conclusão de acordo ao propósito do estudo. & c) Artigos cujo texto não estivesse completamente \\
& disponível online. \\
\hline
\end{tabular}

Fonte: Arquivo pessoal.

Etapa 3: fizeram-se a categorização dos estudos, organização e sumarização das informações dos artigos revisados.

> Etapa 4: avaliação dos estudos na íntegra.

D Etapa 5: foram conduzidas a discussão e a interpretação dos resultados com posterior síntese do conhecimento.

\section{Resultados}

Um total de 58 artigos foram identificados por meio de busca eletrônica em 5 bases de dados. Foram separados todos os artigos que se encontraram na base de dados, combinando os descritores conforme é mostrado na Tabela 1. 
Tabela 1. Estratégia de busca e artigos encontrados por bases de dados.

\begin{tabular}{|c|c|c|}
\hline Base de dados & Palavras-chave & $\begin{array}{l}\text { Números de artigos } \\
\text { encontrados }\end{array}$ \\
\hline $\begin{array}{l}\text { LILACS, MEDLINE, BBO (VIA BVS), } \\
\text { SCIELO E PUBMED }\end{array}$ & $\begin{array}{l}\text { (Rugoscopia OR Rugoscopy) AND (Palatina OR } \\
\text { Palatal) }\end{array}$ & 47 \\
\hline $\begin{array}{l}\text { LILACS, MEDLINE, BBO (VIA BVS), } \\
\text { SCIELO E PUBMED }\end{array}$ & Palatoscopia OR Palatoscopy & 8 \\
\hline $\begin{array}{l}\text { LILACS, MEDLINE, BBO (VIA BVS), } \\
\text { SCIELO E PUBMED }\end{array}$ & $\begin{array}{l}\text { (Odontologia Legal OR Forensic Dentistry) AND } \\
\text { (Rugoscopia OR Rugoscopy) }\end{array}$ & 3 \\
\hline
\end{tabular}

Fonte: Arquivo pessoal.

A Figura 1 abaixo, demonstra o desenvolvimento da seleção dos artigos para esta revisão.

Figura 1. Diagrama de fluxo para seleção dos artigos nas diferentes fases da revisão.

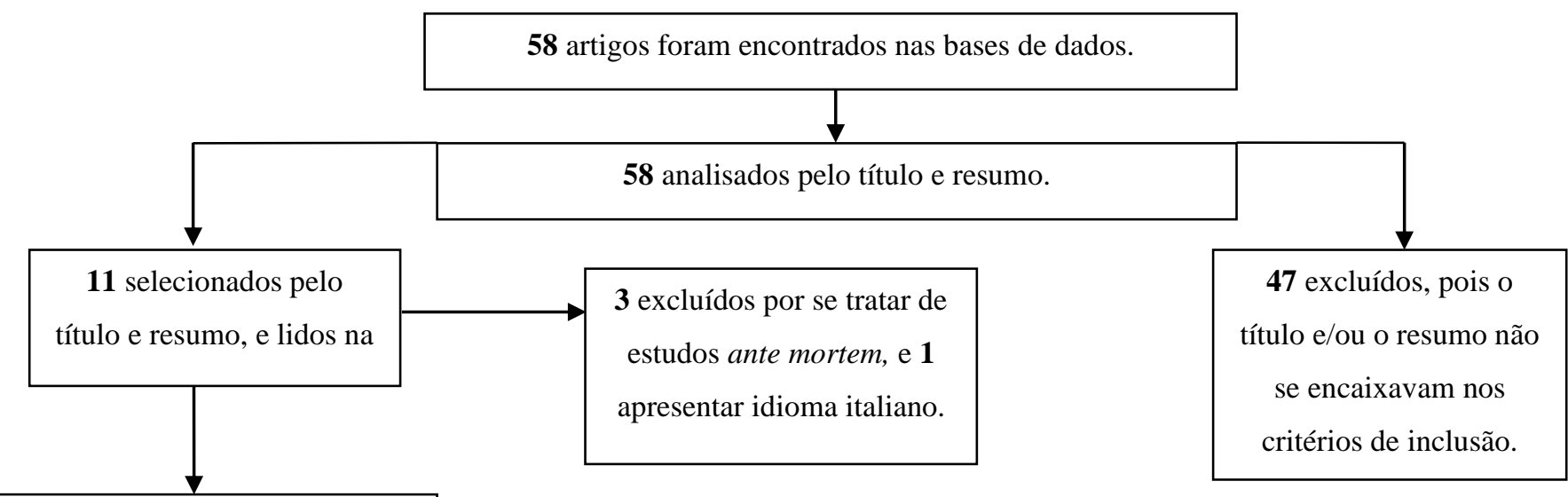

7 atendiam os critérios e foram incluídos na revisão integrativa.

Fonte: Arquivo pessoal.

Na base de dados do Lilacs, foram encontrados 17 artigos; no MedLine, 18 artigos; no PubMed, 21 artigos; na Scielo, 2 artigos, e na BBO, 0 artigos. Destes, de acordo com os critérios de inclusão, foram selecionados 3, 1, 3, 0 e 0 artigos, respectivamente, como exposto no Quadro 2. Os artigos que se repetiram foram considerados em apenas uma das bases de dados. 
Quadro 2. Distribuição das referências incluídas, segundo o título, base de dados, periódico, ano de publicação e país de origem.

\begin{tabular}{|c|c|c|c|c|c|}
\hline $\mathbf{N}^{\mathbf{0}}$ & Título & Base de dados & Periódico & Ano & País de origem \\
\hline 1 & $\begin{array}{l}\text { Rugoscopia, Queiloscopia, Oclusografía } \\
\text { y Oclusoradiografía como métodos de } \\
\text { identificación en odontología forense. } \\
\text { Una revisión de la literatura. }\end{array}$ & LILACS & $\begin{array}{l}\text { Acta Odontológica } \\
\text { Venezolana }\end{array}$ & 2010 & Venezuela \\
\hline 2 & $\begin{array}{lrlrr}\text { Odontología } & \text { Forense } & \text { III: } & \text { Rugas } \\
\text { Palatinas y } & \text { Huellas } & \text { Labiales } & \text { en } \\
\text { Identificación } & \text { Forense. } & & \end{array}$ & LILACS & $\begin{array}{l}\text { Internacional Journal } \\
\text { of Odontostomatology }\end{array}$ & 2014 & Espanha \\
\hline 3 & $\begin{array}{l}\text { Forensic dentistry in human } \\
\text { identification: } \text { A review of the literature. }\end{array}$ & MEDLINE & $\begin{array}{l}\text { Journal of Clinical and } \\
\text { Experimental Dentistry }\end{array}$ & 2014 & Espanha \\
\hline 4 & $\begin{array}{l}\text { Palatal Rugae Patterns in Edentulous } \\
\text { Cases, Are They A Reliable Forensic } \\
\text { Marker? }\end{array}$ & PUBMED & $\begin{array}{l}\text { International Journal of } \\
\text { Biomedical Science: } \\
\text { IJBS }\end{array}$ & 2015 & Índia \\
\hline 5 & $\begin{array}{l}\text { Utilização da rugoscopia palatina para } \\
\text { identificação de corpo carbonizado - } \\
\text { relato de caso pericial. }\end{array}$ & LILACS & $\begin{array}{l}\text { Revista Brasileira de } \\
\text { Odontologia Legal }\end{array}$ & 2016 & Brasil \\
\hline 6 & $\begin{array}{l}\text { Sex determination in forensic } \\
\text { identification, a review }\end{array}$ & PUBMED & $\begin{array}{l}\text { Journal of Forensic } \\
\text { Dental Sciences }\end{array}$ & 2018 & Índia \\
\hline 7 & $\begin{array}{l}\text { Reliability of palatal rugoscopy for } \\
\text { sexual dimorphism in forensic dentistry: } \\
\text { a systematic literature review and meta- } \\
\text { analysis. }\end{array}$ & PUBMED & $\begin{array}{l}\text { Archives of Oral } \\
\text { Biology }\end{array}$ & 2019 & Brasil \\
\hline
\end{tabular}

Fonte: Arquivo pessoal.

No Quadro 3, mostra-se que dos estudos selecionados, 04 (quatro) artigos trataram de uma revisão de literatura do tipo narrativa; 01 (um) artigo trata-se de uma revisão de literatura do tipo sistemática e meta-análise; 01 (um) artigo discute sobre uma revisão de relatos de casos periciais e 01 (um) trata-se de um relato de caso pericial. Em relação a população estudada, com exceção das revisões de literatura, os outros artigos estavam relacionados a cadáveres carbonizados e vítimas de acidentes ou homicídios, e suspeitos de roubo, estupro e outros. 
Quadro 3. Descrição dos estudos incluídos na pesquisa, segundo o tipo de estudo, população estudada e principais resultados.

\begin{tabular}{|c|c|c|c|}
\hline $\mathbf{N}^{\mathbf{0}}$ & Tipo De Estudo & População Estudada & Principais Resultados \\
\hline 1 & $\begin{array}{l}\text { Revisão de Literatura } \\
\text { Narrativa }\end{array}$ & $\begin{array}{l}\text { Literatura sobre os métodos } \\
\text { utilizados pela Odontologia } \\
\text { Legal. }\end{array}$ & $\begin{array}{l}\text { A rugoscopia palatina é vista por esse autor como um } \\
\text { método de identificação humana que leva em } \\
\text { consideração a classificação das rugas palatinas, porém } \\
\text { apresenta algumas situações que dificultam a } \\
\text { identificação por esse método, como: modificações na } \\
\text { altura das cristas, presença de rugas planas ou pouco } \\
\text { acentuadas e a ausência de padrões rugoscópicos } \\
\text { simples. No entanto, outros elementos podem ser } \\
\text { utilizados para auxiliar na identificação como, a papila } \\
\text { incisiva, a forma da rafe palatina e tórus palatino (se } \\
\text { estiver presente). }\end{array}$ \\
\hline 2 & $\begin{array}{l}\text { Revisão de Relatos de } \\
\text { Caso }\end{array}$ & $\begin{array}{lcc}\text { Vítimas de } & \text { acidentes } & \text { ou } \\
\text { homicídios; } & \text { suspeitos } & \text { de } \\
\text { roubo, estupro e outros. } & \end{array}$ & $\begin{array}{l}\text { A rugoscopia palatina representa sem dúvida uma } \\
\text { grande fonte de informação. No entanto, a } \\
\text { possibilidade de distorções originadas por processos } \\
\text { distróficos, reabsorvíveis ou inflamatórios na região } \\
\text { palatina expõe o método a sérios dilemas técnicos e } \\
\text { processuais. Nesse estudo foi detectado apenas } 3 \\
\text { investigações originais que utilizavam dessa técnica de } \\
\text { identificação em uma situação controlada, e em uma } \\
\text { dessas investigações apenas } 79 \% \text { de certeza foi obtido } \\
\text { na comparação das documentações com o achado post } \\
\text { mortem. Situações como perdas ósseas, rugas pouco } \\
\text { demarcadas, inconsistência entre observadores, } \\
\text { técnicas defeituosas de impressão das rugas e } \\
\text { vazamento do gesso, foram utilizadas como } \\
\text { explicações para o baixo percentual de comparação. } \\
\text { Observaram ainda que a comparação das rugas } \\
\text { impressas na prótese de uma vítima e a avaliação intra- } \\
\text { oral nunca serão as únicas evidências para uma } \\
\text { identificação positiva. Afirmam ainda que, as } \\
\text { descobertas da literatura são conflituosas e } \\
\text { insuficientes e que a maioria dos estudos foram } \\
\text { realizados com um pequeno número de amostras. }\end{array}$ \\
\hline 3 & $\begin{array}{l}\text { Revisão de literatura } \\
\text { Narrativa }\end{array}$ & $\begin{array}{l}\text { Literatura publicada nos } \\
\text { últimos } 5 \text { anos sobre o papel } \\
\text { da odontologia } \\
\text { identificação humana. }\end{array}$ & $\begin{array}{l}\text { As rugas palatinas têm sido estudadas como um } \\
\text { método de identificação comparável com as impressões } \\
\text { digitais. No entanto, observaram que as rugas sofrem } \\
\text { modificações na adolescência, com um aumento } \\
\text { acentuado no número de rugas após os } 35 \text { anos de } \\
\text { idade ou decréscimo após os } 23 \text { anos de idade. Ainda }\end{array}$ \\
\hline
\end{tabular}




\begin{tabular}{|c|c|c|c|}
\hline & & & $\begin{array}{l}\text { observaram que existem } 3 \text { situações que complicam a } \\
\text { identificação por meio das rugas palatinas: } \\
\text { modificações na altura das rugas, presença de rugas } \\
\text { planas ou pouco acentuadas e a ausência de padrões } \\
\text { rugoscópicos simples. No entanto, outros elementos } \\
\text { podem suplementar o estudo das rugas palatinas como } \\
\text { a papila incisiva, a forma da rafe palatina, e o tórus } \\
\text { palatino (quando presente). Além disso, a porcentagem } \\
\text { de precisão da identificação através da rugoscopia } \\
\text { palatina pode ser de } 94 \% \text {. }\end{array}$ \\
\hline 4 & $\begin{array}{l}\text { Revisão de Literatura } \\
\text { Narrativa }\end{array}$ & $\begin{array}{l}\text { Literatura sobre a importância } \\
\text { das rugas palatinas em caso de } \\
\text { edêntulismo. }\end{array}$ & $\begin{array}{l}\text { As rugas palatinas têm sido consideradas relevantes } \\
\text { para a identificação humana devido a sua estabilidade } \\
\text { sendo equivalente as impressões digitais, as quais são } \\
\text { únicas para cada pessoa. A identificação pela ruga } \\
\text { palatina pode ser enganada pela pobre demarcação das } \\
\text { eminências rugais no caso do edentulismo; em casos de } \\
\text { rugas severamente baixas; por mudanças na altura } \\
\text { palatina por atrofia do arco alveolar após extrações } \\
\text { dentárias; padrões rugoscópicos simples como rugas } \\
\text { em linha reta. }\end{array}$ \\
\hline 5 & Relato de Caso Pericial & Cadáver carbonizado. & $\begin{array}{l}\text { A rugoscopia palatina, com finalidade forense, é } \\
\text { frequentemente estudada na literatura odontológica no } \\
\text { sentido de comprovar a sua unicidade e imutabilidade. } \\
\text { Entretanto, sabe-se que em decorrência dos } \\
\text { traumatismos e dos processos de decomposição, a } \\
\text { mucosa palatina que contém as rugosidades geralmente } \\
\text { fica comprometida, inviabilizando uma análise } \\
\text { rugoscópica, o que não aconteceu no caso em questão. } \\
\text { Em relação à praticabilidade, verifica-se que a análise } \\
\text { rugoscópica propicia um exame pericial simples, } \\
\text { rápido e de baixo custo, mas que deve ser realizada por } \\
\text { um profissional da Odontologia, devidamente treinado. } \\
\text { Uma das principais desvantagens do uso da análise da } \\
\text { rugoscopia palatina na identificação odontolegal } \\
\text { consiste na dificuldade em se obter dados ante mortem } \\
\text { para comparação com os dados post mortem, pois os } \\
\text { exames que permitem a visualização das rugosidades } \\
\text { não são exames de rotina nos atendimentos clínicos } \\
\text { odontológicos. }\end{array}$ \\
\hline
\end{tabular}




\begin{tabular}{|c|c|c|c|}
\hline 6 & $\begin{array}{l}\text { Revisão de Literatura } \\
\text { Narrativa }\end{array}$ & $\begin{array}{l}\text { Literatura exibida entre } 1950 \text { e } \\
2015 \text { sobre os diferentes } \\
\text { métodos de determinação } \\
\text { sexual. }\end{array}$ & $\begin{array}{l}\text { A rugoscopia palatina é vista como um método } \\
\text { suplementar para determinação do sexo de um } \\
\text { indivíduo através da presença de próteses dentárias } \\
\text { rotuladas com o nome do indivíduo ou outra forma de } \\
\text { identificação (número de telefone, endereço e etc.). } \\
\text { Além disso, discute sobre a comparação dos padrões } \\
\text { rugoscópicos em homens e mulheres através de uma } \\
\text { revisão bibliográfica. }\end{array}$ \\
\hline 7 & $\begin{array}{l}\text { Revisão de } \\
\text { Sistemática e } \text { Meta- } \\
\text { Análise }\end{array}$ & $\begin{array}{l}\text { Literatura buscada online e } \\
\text { manual sobre o poder e } \\
\text { confiabilidade da rugoscopia } \\
\text { palatina no dimorfismo sexual. }\end{array}$ & $\begin{array}{l}\text { A rugoscopia palatina é um procedimento técnico } \\
\text { utilizado na rotina da odontologia legal para auxiliar a } \\
\text { identificação humana. Tanto na abordagem } \\
\text { comparativa quanto na reconstrutiva da identificação } \\
\text { humana a rugoscopia se mostrou útil como uma } \\
\text { ferramenta de identificação humana baseada } \\
\text { principalmente na sua apresentação de padrões } \\
\text { distintos das rugas palatinas. O estudo de meta-análise } \\
\text { realizado mostrou que a ruga palatina alcançou valores } \\
\text { de precisão, assim como alta taxa de sensibilidade e } \\
\text { especificidade para o dimorfismo sexual. No entanto, } \\
\text { recomenda que esse método seja usado apenas em } \\
\text { combinação com outros métodos para dimorfismo } \\
\text { sexual já consolidados na literatura científica, pois as } \\
\text { limitações encontradas em cada estudo sugerem que a } \\
\text { rugoscopia carece de confirmação baseada em } \\
\text { evidência para aplicação confiável como única } \\
\text { ferramenta para o dimorfismo sexual na prática. Além } \\
\text { disso, limitações relacionadas ao método como: a } \\
\text { realização de moldagens e obtenção de modelos de } \\
\text { gesso manualmente podem distorcer a forma e tamanho } \\
\text { da ruga palatina. No mesmo contexto, a análise das } \\
\text { rugas com lápis e lupa mostra-se mais subjetivo do que } \\
\text { quando realizada virtualmente. }\end{array}$ \\
\hline
\end{tabular}

Fonte: Arquivo pessoal.

\section{Discussão}

Os estudos incluídos na pesquisa foram unanimes em destacar a importância da rugoscopia palatina, sempre trazendo aspectos relevantes para seu uso. Ao se referirem a mesma, vários autores corroboraram sobre a possibilidade de obtenção de muitas informações sobre uma determinada vítima (Carjevschi, 2010; Fonseca, Cantín, \& Lucena, 2014; Ata-Ali \& Ata-Ali, 2014; Poojya et al., 2015), sendo inclusive método comparável ao método datiloscópico devido a estabilidade e unicidade que as rugas palatinas apresentam (Ata-Ali \& Ata-Ali, 2014; Poojya et al., 2015). 
Um relato de caso pericial demonstrou a viabilidade do emprego da rugoscopia palatina na identificação de um corpo carbonizado de um indivíduo não identificado para a fim de se obter a causa mortis. Foram apresentados, juntamente com o prontuário do suspeito, os modelos de gesso relativos à documentação ortodôntica, sendo possível a visualização nos mesmos, do palato duro e das rugas palatinas. Verificaram, por meio de comparação entre os registros anteriores e posteriores a morte, que se tratava daquele individuo, concluindo pela viabilidade da técnica, inclusive justificando que seu uso de forma isolada ou em conjunto com outros métodos de identificação, podem ser base para a identificação de cadáveres (Argollo et al., 2017). No entanto, é importante considerar que se trata de um relato de caso, o que não deve ser ampliado indiscriminadamente para todos os tipos de casos, ou seja, o odontolegista deve ter ponderação em suas indicações, pois seu uso deve ser sempre baseados em evidencias consolidadas, o que promoverá segurança quando utilizada como único método de identificação de uma vítima.

Ao serem consideradas as limitações sobre a utilização da técnica da rugoscopia palatina, a sua precisão de identificação foi destacada, sendo mensurada em torno de 94\% (Ata-Ali \& Ata-Ali, 2014) e em 79\% quando levado em consideração o uso da comparação das documentações ante mortem com o achado post mortem (Fonseca, Cantín, \& Lucena, 2014).

A rugoscopia também é um método de identificação humana suplementar ou auxiliar para a determinação do sexo da vítima. Em revisão de literatura, foi salientado que quando a identificação de um indivíduo torna-se dificultosa por outros métodos, uma alternativa a ser empregada é a rugoscopia palatina, desde que haja material para se proceder a comparações. Os autores discutem, que apesar das rugas palatinas serem protegidas por sua posição interna na cabeça, alguns eventos podem contribuir para que ocorram mudanças no seu padrão, tendo como exemplos o trauma, sucção extrema do dedo na infância, pressão persistente com tratamento ortodôntico e uso de próteses. Foi evidenciado que o uso de próteses dentárias rotuladas com o nome do indivíduo ou outra forma de identificação como sexo, endereço ou número de telefone podem ser úteis na identificação da vítima (Nagare et al., 2018).

Após revisão sistemática relativa à relação da rugoscopia palatina e o dimorfismo sexual, a qual resultou 263 estudos, sendo que 08 preencheram os critérios de inclusão, os autores verificaram que o método pareceu ter este poder discriminante. Entretanto, além de observarem as limitações relativas a cada estudo analisado, bem como uma expressiva heterogeneidade entre ele, concluiu que, sob o viés do dimorfismo sexual, há necessidade de maiores confirmações baseadas em evidências (Andrade et al., 2019).

Estudos convergem no que refere as limitações do método rugoscopia palatina como uma forma de identificação humana, justificando que modificações na altura das cristas, presença de rugas planas ou pouco acentuadas e a ausência de padrões rugoscópicos simples podem dificultar o processo de identificação da vítima, mas que mesmo com a presença dessas características, existem outros elementos como a papila incisiva, a forma da rafe palatina e a presença de tórus palatino, os quais podem auxiliar na identificação (Carjevschi, 2010; Ata-Ali \& Ata-Ali, 2014).

Outro estudo demonstra que algumas dessas limitações (modificações na altura das rugas, rugas severamente baixas, ausência de padrões rugoscópicos simples - linha reta) também estão presentes em casos de edentulismo, e que além destas, situações de mudança da altura palatina devido atrofia do arco alveolar, após extrações dentárias, também podem causar problemas de identificação (Poojya et al., 2015).

Diversas situações inerentes ao tecido estudado e situações inerentes a técnica podem dificultar a identificação humana utilizando como método a rugoscopia palatina (Fonseca, Cantín, \& Lucena, 2014). Nas inerentes ao tecido, temos que distorções originadas por processos distróficos, reabsorvíveis, inflamatórios, perdas ósseas e rugas pouco demarcadas expõem o método a sérios dilemas técnicos e processuais; Já nas situações inerentes a técnica, a inconsistência entre observadores, 
técnicas defeituosas de impressão das rugas palatinas e de vazamento do gesso são situações que podem causar problemas na identificação de uma vítima (Andrade et al., 2019).

Quanto as formas de examinar as rugas palatinas, estas se apresentam como várias, sendo a inspeção intraoral sugerida como o método mais utilizado, pela sua facilidade e por ser mais barato. Entretanto, o estudo das impressões e fotografias tem sido entendido como mais detalhado. Pode-se usar, inclusive duas fotos tiradas da mesma câmera e sobrepô-las para proceder ao exame usando softwares de computador. As próteses totais, inclusive, podem ser fortes aliadas à identificação, em face da presença da caracterização das rugas na parte mucosa, sendo este um método suplementar constatado em casos periciais realizados. Entretanto, as fotografias e impressões das rugas palatinas ainda têm sido considerados métodos mais detalhados e precisos, sendo guias confiáveis de investigação (Fonseca, Cantín, \& Lucena, 2014; Poojya et al., 2015; Nagare et al., 2018).

\section{Considerações Finais}

Podemos concluir que a rugoscopia palatina tem se apresentado na literatura científica sendo um método útil como guia na identificação humana, normalmente complementar a outros métodos de identificação mais consolidados, e ainda com possibilidade de transmitir diversas informações, tais como em situações de investigação do sexo. Além disso, foi verificado que ele não deve ser utilizado como único método de identificação da vítima, posto que também apresenta várias limitações inerentes a técnica de impressão das rugas palatinas ou ao próprio tecido, as quais podem dificultar ou mesmo inviabilizar o procedimento identificatório.

Pode-se observar também, que a literatura acerca deste tema, considerando todos os critérios de inclusão e exclusão elencados para a busca da literatura neste estudo, é extremamente restrita para afirmar a utilidade da rugoscopia palatina como a única forma de identificar uma vítima, e por isso, necessita, em trabalhados futuros, de um maior enriquecimento realizando pesquisas post mortem para verificar sua confiabilidade na identificação humana com o propósito de torna-la mais uma ferramenta para esse processo.

\section{Referências}

Adisa, A. O., Kolude, B., \& Ogunrinde, T. J. (2014). Palatal rugae as a tool for human identification. Nigerian journal of clinical practice, 17(5), 641-643.

Alves, A. V. N., \& Vasconcellos, R. C. D. (2016). Identificação humana em desastres em massa através da odontologia forense. Trabalho de Conclusão de Curso, Centro Universitário São Lucas.

Andrade, R. N. M., de Andrade Vieira, W., de Macedo Bernardino, Í., Franco, A., \& Paranhos, L. R. (2019). Reliability of palatal rugoscopy for sexual dimorphism in forensic dentistry: A systematic literature review and meta-analysis. Archives of oral biology, 97, 25-34.

Argollo, S. D. P., Argollo, B. P., Argollo, P. A. N. D., \& Marques, J. A. M. (2017). Utilização da rugoscopia palatina para identificação de corpo carbonizado relato de caso pericial. Rev. Bras. Odontol. Leg. RBOL, 107-113.

Ata-Ali, J., \& Ata-Ali, F. (2014). Forensic dentistry in human identification: A review of the literature. Journal of clinical and experimental dentistry, 6(2), e162.

Babaji, P., Jalal, S. A., \& Kamalaksharappa, S. K. (2018). Evaluation of palatal rugae pattern in identification and sex determination in Indian children. Pesquisa Brasileira em Odontopediatria e Clínica Integrada, 18(1), 3944.

Barbieri, A. A., Scoralick, R. A., Naressi, S. C., Moraes, M. E., Daruge Jr, E., \& Daruge, E. (2013). The evidence of the rugoscopy effectiveness as a human identification method in patients submitted to rapid palatal expansion. Journal of forensic sciences, 58, S235-S238.

Carjevschi Moses, G. (2010). Rugoscopia, queiloscopia, oclusografía y oclusoradiografía como métodos de identificación en odontología forense: una revisión de la literatura. Acta odontol. venez.

Castro-Silva, I. I., Silva, O. M. L. D., \& Veiga, B. M. C. (2014). Uso da rugoscopia palatina como ferramenta biométrica: um estudo populacional em NiteróiRJ, Brasil. Revista de Odontologia da UNESP, 43(3), 203-208.

Correia, A. D. M., Barbosa, D. D. S., Alcântara, J. A. D. S., Fontenele, E. H. L., \& Bezerra, T. P. (2019). Importância do registro das ausências dentais para a identificação humana: relato de caso. Rev. Bras. Odontol. Leg. RBOL, 82-89. 
Eboh, D. E. (2012). Palatal rugae patterns of urhobos in Abraka, South-Southern Nigeria. Int J Morphol, 30(2), 709-713.

Fonseca, G. M., Cantín, M., \& Lucena, J. (2014). Odontología Forense III: rugas palatinas y huellas labiales en identificación forense. International journal of odontostomatology, 8(1), 29-40.

Garbin, C. A. S., Amaral, M. A., \& da Silva Greghi, R. S. (2017). Análise e classificação da rugosidade palatina em uma população brasileira. Revista Brasileira de Odontologia Legal, 4(3).

Gomes, I. D. S. A., Alves, L. T., Fontes, N. M., de Medeiros Batista, M. I. H., Carvalho, A. A. T., \& Paulino, M. R. (2019). Importância da identificação humana através de marcas de mordida: uma revisão da literatura. Derecho y Cambio Social, (57), 722-737.

Junior, E. F., \& de Moura, L. C. L. (2014). A importância dos arcos dentários na identificação humana. Revista Brasileira de Odontologia, 71(1), 22.

Lima, M. V. D. F. N., Costa, G. M., da Silva, V. B., do Nascimento, M. R., de Moraes, H. H., \& de Souza Lucena, E. E. (2016). Verificação da praticabilidade e da unicidade na queiloscopia e na palatoscopia como métodos de identificação humana. Revista Brasileira de Odontologia Legal, 3(1).

Matute, G. A. R. (2015). La rugoscopia palatina forense como método de identificación humana a través del análisis comparativo. Revista Científica de la Escuela Universitaria de las Ciencias de la Salud, 2(1), 37-42.

Miranda, R. F., Lima, L. N. C., Tinoco, R. L. R., Rabello, P. M., Costa, A. P. S., \& Júnior, E. D. (2011). Palatal rugoscopy as a method of human identification. Odonto, 19(38), 71-79.

Modesto, T. D. O. P., \& Junior, E. F. (2017). Identificação humana através da Rugoscopia Palatina. Revista interdisciplinar de direito, 11(2).

Nagare, S. P., Chaudhari, R. S., Birangane, R. S., \& Parkarwar, P. C. (2018). Sex determination in forensic identification, a review. Journal of forensic dental sciences, $10(2), 61$.

Oliveira, G. S., \& Marques, J. A. M. (2015). Uso da tecnologia de impressão tridimensional na rugoscopia palatina. Revista Brasileira de Odontologia Legal, 2(2).

Olivo, J. L., Ricardo, J. H., \& García, K. C. (2014). Análisis de la forma y distribución de rugas palatinas en la identificación humana. Medicina Legal de Costa Rica, 31(1), 23-30.

Poojya, R., Shruthi, C. S., Rajashekar, V. M., \& Kaimal, A. (2015). Palatal rugae patterns in edentulous cases, are they a reliable forensic marker?. International journal of biomedical science: IJBS, 11(3), 109.

Santos, K. C., Fernandes, C. M. S., \& da Costa Serra, M. (2011). Evaluation of a digital methodology for human identification using palatal rugoscopy. Brazilian Journal of Oral Sciences, 10(3), 199-203.

Tornavoi, D. C., \& da Silva, R. H. A. (2010). Rugoscopia palatina e a aplicabilidade na identificação humana em odontologia legal: revisão de literatura. Saúde, Ética \& Justiça, 15(1), 28-34.

Whittemore, R., \& Knafl, K. (2005). The integrative review: updated methodology. Journal of advanced nursing, 52(5), 546-553. 